\title{
Lipid Nanoparticles Enabling Gene Therapies: From Concepts to Clinical Utility
}

\author{
Jayesh A. Kulkarni, ${ }^{1}$ Pieter R. Cullis, ${ }^{1}$ and Roy van der Meel ${ }^{1,2}$
}

Genetic drugs based on RNA or DNA have remarkable therapeutic potential as virtually any disease can be treated by silencing a pathological gene, expressing a beneficial protein, or by editing defective genes. However, therapies based on nucleic acid polymers require sophisticated delivery systems to deliver these macromolecules to the interior of target cells. In this study, we review progress in developing nonviral lipid nanoparticle (LNP) delivery systems that have attractive properties, including ease of manufacture, reduced immune responses, multidosing capabilities, larger payloads, and flexibility of design. LNP systems represent the most advanced delivery systems for genetic drugs as it is expected that an LNP-short interfering RNA (siRNA) formulation will receive clinical approval from the Food and Drug Administration (FDA) in 2018 for treatment of the hereditary condition transthyretin-mediated amyloidosis, a fatal condition for which there is currently no treatment. This achievement is largely due to the development of optimized ionizable cationic lipids, arguably the most important factor in the clinical success of LNP-siRNA. In addition, we highlight potential LNP applications, including targeting tissues beyond the liver and therapeutic approaches based on messenger RNA or Clustered Regularly Interspaced Short Palindromic Repeats/Cas.

Keywords: gene therapy, genetic drugs, drug delivery, nucleic acid, lipid nanoparticle, ionizable cationic lipid

\section{Introduction}

G ENETIC INFORMATION FLOWS from genomic DNA to functional protein products through a messenger RNA (mRNA) intermediate. Expression of the protein product is modulated by short sequences of RNA through RNA interference (RNAi). As a result, genetic drugs based on plasmid DNA (pDNA), mRNA, and short interfering RNA (siRNA) are potentially able to treat many diseases by targeting the root cause rather than blocking downstream signaling pathways or treating symptoms. Furthermore, the development of geneediting approaches using Clustered Regularly Interspaced Short Palindromic Repeats (CRISPR) has facilitated the replacement of dysfunctional genes with the wild-type allele in a straightforward manner, potentially enabling correction of hereditary disorders. The greatest remaining barrier to enabling clinical use of genetic drugs is effective intracellular delivery into target tissues in vivo [1-5]. Viral vectors suffer from immunogenicity, manufacturing, and other concerns, whereas nonviral vectors historically have had toxicity and potency issues. However, the recent successful Phase III trial of a lipid nanoparticle (LNP) formulation of siRNA to treat transthyretin (TTR)-induced amyloidosis suggests that nonviral vectors are starting to overcome the delivery barrier [6].
A key advance has been identification and incorporation of an optimized ionizable cationic lipid in the LNP-siRNA systems, a lead example being heptatriaconta-6,9,28,31-tetraen-19-yl 4(dimethylamino)butanoate (DLin-MC3-DMA or MC3) [7,8]. Incorporation of MC3 in LNP-siRNA systems has led to highly potent gene silencing systems that are effective for silencing genes in the liver at dose levels as low as $5 \mu \mathrm{g}$ siRNA/ $\mathrm{kg}$ in murine models.

Alnylam ${ }^{\circledR}$ Pharmaceuticals' formulation, known as Patisiran, met all primary and secondary endpoints, and showed robust and sustained knockdown of TTR [9]. Submission of a New Drug Application to the Food and Drug Administration (FDA) was recently completed [10] and the expected approval of Patisiran in 2018 will mark the first successful systemic RNAi treatment for patients. The European Medicines Agency (EMA) recently accepted the marketing authorization application for Patisiran [11]. In addition, there are a number of LNP formulations of siRNA and other genetic drugs currently undergoing clinical evaluation for treatment of various diseases, including cancer and viral infections (Table 1).

Lipid-based drug delivery systems represent a technology with a large knowledge base and well-understood design principles. There are now $10 \mathrm{LNP}$ drugs that have received

\footnotetext{
${ }^{1}$ Department of Biochemistry and Molecular Biology, University of British Columbia, Vancouver, British Columbia, Canada.

${ }^{2}$ Department of Clinical Chemistry and Haematology, University Medical Center Utrecht, Utrecht, the Netherlands.
} 


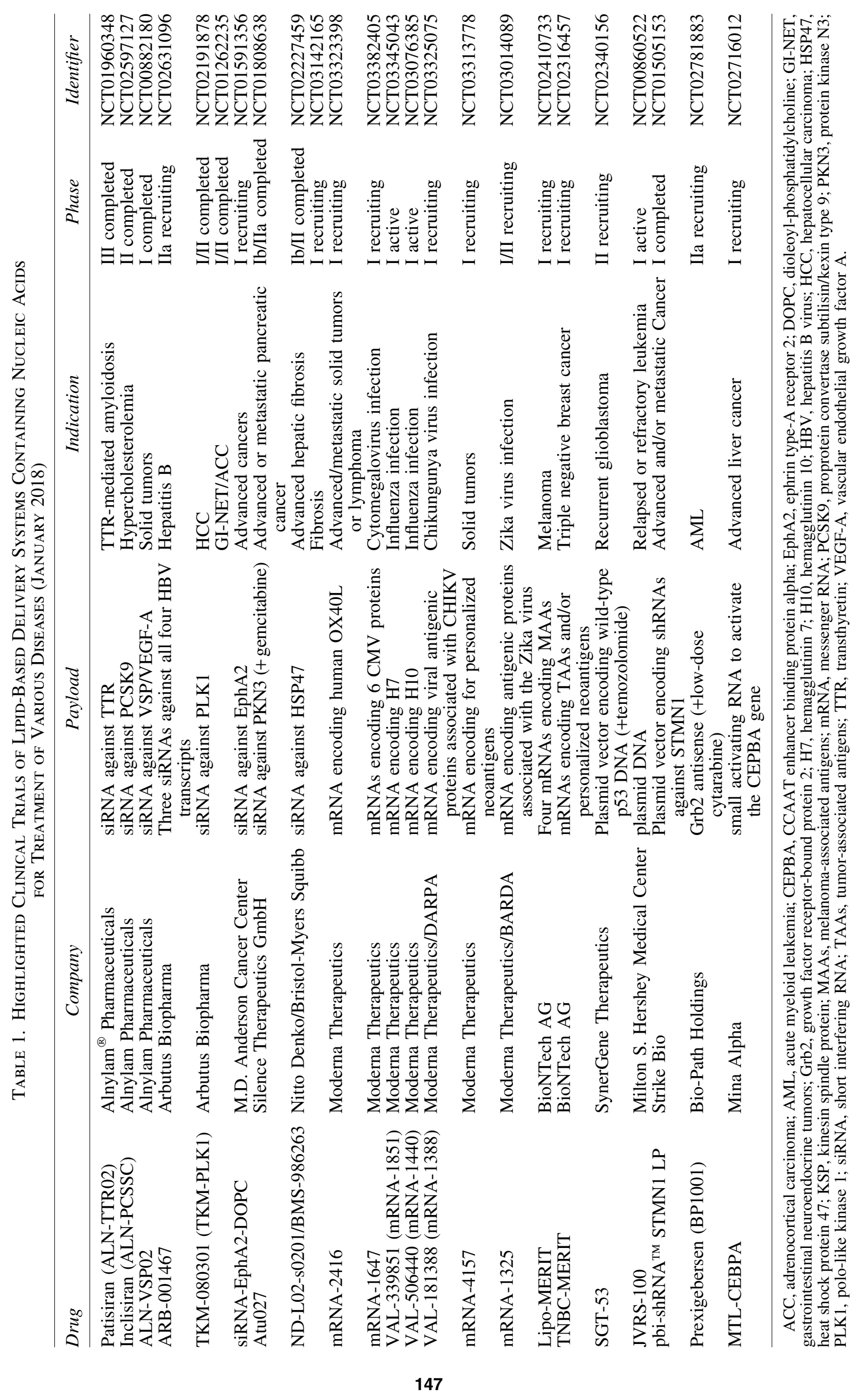


regulatory approval for delivery of small molecule drugs [12]. In this study, we review the challenges involved in extending LNP technology to nucleic acid polymers, the critical factors that have enabled progress to this point, and the remarkable way in which LNP technology developed for siRNA can also be applied to much larger payloads such as mRNA and pDNA.

\section{Barriers to Gene Therapies Based on Nucleic Acid Polymers}

Initial research in the 1990s focused on the delivery of DNA, with the aim to induce expression of a gene of interest in target cells. The half-life of functional pDNA in mouse whole blood (in vitro) was shown to be $\sim 10 \mathrm{~min}$, and even shorter in vivo upon intravenous (i.v.) administration [13] where rapid and extensive hepatic accumulation in nonparenchymal cells were observed. This elimination from circulation was largely a result of serum nuclease activity and represents the first major barrier to delivery of nucleic acid therapeutics. The activation of innate immunity represents a second impediment [14]. In the case of mRNA and siRNA, this immune response can be partially circumvented through chemical modification of the nucleic acids [15]. On the other hand, pDNA, which is purified from biological sources rather than synthesized, is less amenable to such modifications. It also suffers from an increased likelihood of pyrogens (endotoxins) being present in the raw material. However, even if nucleic acids are heavily modified to overcome serum nuclease activity and immune stimulation, they are still subject to a third barrier, renal filtration (efficient filtration of particles smaller than $\sim 6 \mathrm{~nm}$ ) $[16,17]$. Studies into the pharmacokinetics of oligonucleotides showed more than $50 \%$ of the dose excreted in urine $[18,19]$. These observations suggest that the utility of unmodified nucleic acid polymers as therapeutics is limited due to their poor bioavailability. Modifications to improve the activity of nucleic acids without delivery vectors have been reviewed elsewhere [20].

Attempts have been made to improve the bioavailability of nucleic acid drugs by predosing with negatively charged polymers such as dextran sulfate [13]. While this improves the pharmacokinetics, the nucleic acids still do not preferentially accumulate at target sites. Even if accumulation is achieved, the physicochemical properties of nucleic acids (bulky size and high charge density) represent a fourth barrier as they prevent diffusion across the cell plasma membrane. Internalization through endocytosis presents problems as the endocytic pathway ends in fusion with the lysosome, where the nucleic acid polymer would be degraded, requiring escape from the endosome to the cell cytoplasm for the DNA or RNA vector to be active. In the case of pDNA and CRISPR therapeutics, there is the subsequent barrier of entering the nucleus for activity [21] as such transport does not occur through passive diffusion unless cell division occurs where the nuclear membrane is temporarily compromised [22].

To achieve delivery across these barriers, many types of systems have been explored [2-4]. In this study, we focus on lipid-based delivery systems, and the first demonstration of lipid-based transfection was an in vitro study by Felgner $e$ al. using the positively charged lipid, $N$-[1-(2,3-dioleyloxy) propyl]- $N, N, N$-trimethylammonium chloride (DOTMA) [23]. Compared to techniques such as calcium phosphate or DEAE- dextran-mediated transfection, "lipofection" was found to be between 5- to 100-fold more effective. This observation started the development of lipid-based vectors for nucleic acid delivery.

\section{Design of LNPs for Delivery of Nucleic Acids}

As noted, LNP formulations of small molecule drugs represent a relatively mature technology. Design features (reviewed elsewhere [12,24]) such as optimal particle sizes (100 nm diameter or less), near $100 \%$ encapsulation efficiencies, robust manufacturing processes, and low surface charge to reduce interactions with serum proteins are also required features for lipid-based delivery systems for nucleic acids. However, different loading strategies and manufacturing techniques are required. These methods must enable efficient and scalable entrapment $(>80 \%)$ of nucleic acid polymer as well as an ability of the resulting LNP to deliver cargo to the cytoplasm of target cells. To achieve encapsulation of negatively charged polymers, cationic lipids are required; these lipids have gone through considerable evolution as detailed below.

\section{Cationic lipids and lipoplexes}

Early efforts to develop LNP systems relied on entrapment through passive methods using neutral (zwitterionic) lipids, which did use electrostatic properties to achieve entrapment. Such formulations displayed poor entrapment efficiencies $(<40 \%)$ [25] and limited transfection potency [26]. The development of DOTMA [23] enabled more efficient entrapment and transfection potency through formation of complexes where DOTMA was mixed with a "helper lipid," dioleoylphosphatidylethanolamine (DOPE), and complexed by mixing with DNA in solution. These complexes, termed "lipoplexes," are unstable and characterized by broad size distributions ranging from the submicron scale to a few microns. Lipoplexes, such as the Lipofectamine ${ }^{\circledR}$ reagent, have found considerable utility for in vitro transfection. However, these first-generation lipoplexes have not proven useful in vivo. The large particle size and positive charge (imparted by the cationic lipid) result in rapid plasma clearance, hemolytic and other toxicities, as well as immune activation [27,28].

\section{Cationic lipids and LNPS}

The first example of an LNP formulation of a genetic drug (pDNA) containing a cationic lipid employed a detergent dialysis technique. The approach comprised mixing of pDNA with cationic lipids (dioctadecyl-dimethyl-ammonium chloride or DODAC) in the presence of a detergent such as octylgluopyranoside (OGP), followed by addition to a solution containing phosphatidylcholine (PC)-lipids and polyethylene glycol (PEG)-lipid in an OGP solution [29]. The final mixture was then dialyzed for $36-48 \mathrm{~h}$ to remove detergent and form nanoparticles. At cationic lipid contents of 6-8 mol\%, entrapment efficiencies of $\sim 30 \%$ were achieved, but replacement of the PC-lipid with DOPE resulted in entrapment efficiencies of $70 \%$. These particles were termed stabilizedplasmid-lipid-particles (SPLP) and displayed unilamellar structure under cryo-TEM with relatively monodisperse size distributions around $70 \mathrm{~nm}[30,31]$. 
SPLP exhibited desired long circulation properties with $t_{1 / 2}$ of $\sim 7 \mathrm{~h}$ in mice compared to $<10 \mathrm{~min}$ for naked pDNA. This enabled $2.5 \%$ of the injected dose to accumulate at a distal tumor over $24 \mathrm{~h}$ [31], resulting in expression of a luciferase reporter in tumor tissue, with very low levels in the liver and spleen [32]. Importantly, the liver toxicity induced by SPLP was significantly lower when compared to lipoplexes generated using the same lipid mixture and lipid-pDNA ratio [31]. At a dose of $30 \mu \mathrm{g}$ DNA, lipoplexes induced a 100-fold increase in levels of liver enzymes alanine aminotransferase (ALT) and aspartate aminotransferase (AST) in serum, while SPLP at a dose of $175 \mu \mathrm{g}$ DNA showed baseline levels of ALT and AST in serum. However, given the low encapsulation efficiency, limited potency, and the fact that the detergent dialysis manufacturing technique is tedious and not scalable, the SPLP approach was not pursued further.

Efficient encapsulation of high levels of nucleic acid polymers in LNP systems intuitively requires high levels of cationic lipids to associate with the negatively charged polymers. However, cationic lipids have inherent limitations for in vivo use. LNPs with a significant surface charge adsorb serum proteins and are rapidly cleared from the circulation [12]. Furthermore, cationic lipids combine with endogenous anionic lipids to form nonlamellar $\mathrm{H}_{\text {II }}$ phase structures [33], thus disrupting cell membranes and accounting partially for their toxicity. Another contribution to toxicity results from the production of reactive oxygen species [34-36]. Cationic lipids, however, are required to achieve efficient loading of nucleic acid polymers into LNPs. To address this problem, ionizable cationic lipids such as 1,2-dioleoyl-3-dimethylammonium propane (DODAP) were developed with apparent $\mathrm{pKa}$ values below 7. LNPs could then be loaded with nucleic acid polymers at $\mathrm{pH}$ values below the $\mathrm{pKa}$ of the ionizable lipid where it is positively charged. However, at physiological $\mathrm{pH}$ values, the LNP would adopt a relatively neutral exterior [37]. Development of lipids such as 1,2-dioleyloxy-N,N-dimethyl-3aminopropane (DODMA) or DODAP (Fig. 1) provided the near-neutral charge in circulation, allowing for significant increases in the circulation half-lives of the particles following i.v. administration [38].

\section{Ionizable Cationic Lipids and Ethanol Loading}

The detergent-dialysis loading process, while effective for producing homogenous LNP, is laborious. Thus, ethanol loading processes were devised for use in combination with ionizable cationic lipids. The first version involved dissolving lipids in ethanol and adding this solution to an aqueous solution of antisense oligonucleotides (ASO) in $\mathrm{pH} 4$ buffer [38]. The resulting particles were then extruded to achieve a homogenous population of $\sim 100 \mathrm{~nm}$ LNP, followed by dialysis to remove solvent and neutralize the $\mathrm{pH}$. This process resulted in entrapment efficiencies of $80 \%$ for a lipid composition of DODAP/DSPC/Cholesterol/PEG-ceramide-C14 $(20 / 25 / 45 / 10 \mathrm{~mol} \%)$. In the next iteration [39], liposomes were initially formed by extrusion and then subjected to ethanol concentrations as high as $40 \%(\mathrm{v} / \mathrm{v})$ in the presence of ASO at acidic $\mathrm{pH}$. The resulting particles displayed entrapment efficiencies as high as $90 \%$ for the ASO and $70 \%$ for pDNA systems. The inclusion of at least $2.5 \mathrm{~mol} \%$ PEG-lipid resulted in stable particles, although up to $10 \mathrm{~mol} \%$ PEGlipid was required to maintain particle size through the incubation.

The final iteration of the ethanol loading process involved the use of a spontaneous vesicle formation process [40] following the Batzri and Korn ethanol injection method [41]. In this process, the desired lipids are dissolved in ethanol at appropriate ratios, while pDNA is dissolved in an acidic buffer. The solutions are then rapidly mixed through a T-junction [42] with an output of $1 \mathrm{~mL} / \mathrm{s}$ where the ethanolic phase contains lipid at a concentration of $\sim 20 \mathrm{mM}$. The resulting nanoparticles were then immediately put through another $\mathrm{T}$ junction to dilute the ethanol concentration. After the first mixing step, the entrapment was determined to be $\sim 60 \%$,
A

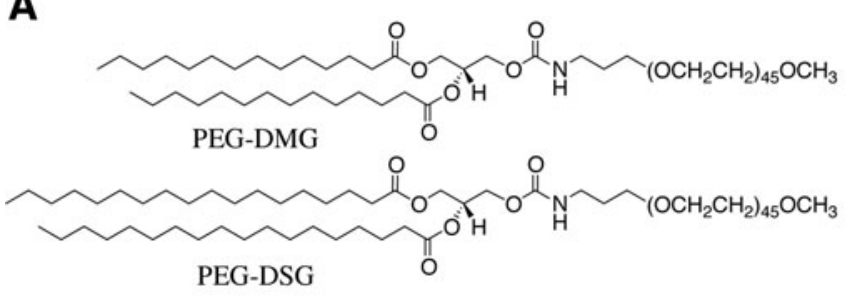

C
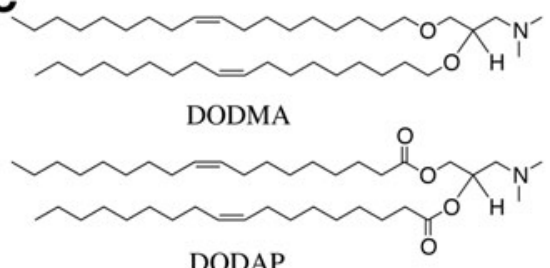

B

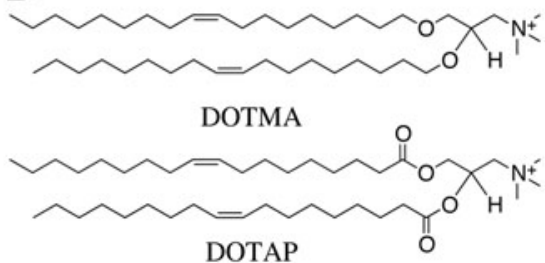

D

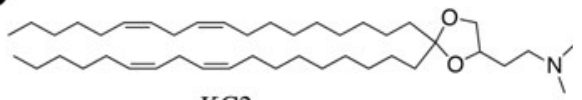

$\mathrm{KC} 2$

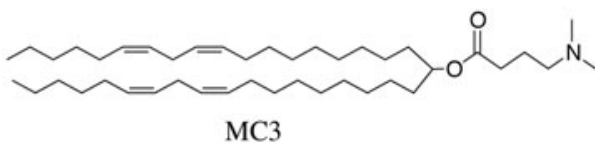

FIG. 1. The evolution of lipids to enable gene silencing in vivo. (A) Diffusible PEG-lipid, PEG-DMG, and persistent PEGlipid, PEG-DSG. (B) Cationic lipids DOTMA and DOTAP. (C) Ionizable cationic lipids DODMA and DODAP. (D) Optimized ionizable cationic lipids KC2 and MC3 for in vivo hepatocyte gene silencing. DODAP, 1,2-dioleoyl-3-dimethylammonium propane; DODMA, 1,2-dioleyloxy-N,N-dimethyl-3-aminopropane; DOTAP, 1,2-dioleoyl-3-trimethylammonium-propane; DOTMA, $N$-[1-(2,3-dioleyloxy) propyl]- $N, N, N$-trimethylammonium chloride; PEG-DMG,(R)-2,3-bis(myristoyloxy)propyl1-[methoxy poly(ethylene glycol)2000] carbamate; PEG-DSG,(R)-2,3-bis(stearoyloxy)propyl-1-[methoxy poly(ethylene glycol)2000] carbamate; PEG, polyethylene glycol. 
increasing to $80 \%-90 \%$ after the second dilution. An improvement to this loading procedure relied on the combination of the two mixing steps into a single step to achieve a final concentration of $25 \%$ ethanol (v/v), although the mixing was performed with a microfluidic mixing device with an output of $2 \mathrm{~mL} / \mathrm{min}$, resulting in an effective 30 -fold reduction in output [43]. This method of diluting lipid-in-ethanol into an aqueous buffer by rapid mixing is now the formulation technique of choice. These methods have been reviewed in detail elsewhere [44]. The method achieves effectively complete encapsulation and has the added advantage of being relatively gentle as required by nucleic acid polymers. Techniques requiring high temperature and high shear forces likely cannot be used.

\section{Ionizable Cationic LNPs for siRNA Delivery}

Application of the ethanol loading technique in combination with ionizable cationic lipids such as DODAP resulted in LNP formulations of ASO that had potential in vivo applications. However, efforts to use LNP systems to improve ASO gene silencing potency had limited success, while immunostimulatory applications had more potential, although this has not yet been exploited [45-47]. The most compelling application, however, was for siRNA. Using the dilinoleoyl version of the ether analogue of DODMA, Dilinoleyl-DMA (DLinDMA), it was found that LNP-siRNA systems resulted in significant gene silencing in the liver following i.v. administration [48]. These authors used the ethanol loading T-junction process and the lipid composition DLinDMA/ cholesterol/DSPC/PEG-lipid (30/48/20/2 mol\%) to formulate LNP ApoB-siRNA and showed dose-dependent silencing of ApoB in the liver $48 \mathrm{~h}$ after a single injection, with maximal silencing of $>90 \%$ in nonhuman primates (cynomolgus monkeys) at doses of 1 or $2.5 \mathrm{mg} / \mathrm{kg}$. Significant reductions in ApoB protein, serum cholesterol, and low-density lipoprotein levels were observed as early as $24 \mathrm{~h}$ after treatment and lasted for nearly 2 weeks at the highest dose.

The type of PEG-lipid used in the ApoB silencing study was termed "diffusible" PEG-lipids, as in the presence of a lipid sink (such as serum lipoproteins), the PEG-lipids were able to diffuse out of the nanoparticle in times on the order of minutes and expose the surface of the LNP [32]. Diffusible PEG-lipids had shorter acyl chains (C14; (R)-2,3-bis(myristoyloxy)propyl1-(methoxy poly(ethylene glycol)2000) carbamate, abbreviated as PEG-DMG) than the persistent PEG-lipids (C18; (R)-2,3bis(stearyloxy)propyl-1-(methoxy poly(ethylene glycol)2000 carbamate, abbreviated as PEG-DSG) that have extended residence times used for LNP systems where long circulation lifetimes are required (Fig. 1). The use of the diffusible PEG-lipids to dissociate the PEG coating renders the LNP transfection competent and contributed significantly to improving transfection. The evolution of these PEG-lipids has been reviewed elsewhere [49], but it is important to note that the PEG-lipid is essential to maintaining the size and stability of the LNP before administration. Furthermore, the use of diffusible PEG lipids mitigates against an immune response to LNP-associated PEG as they do not remain associated with the LNP [50].

\section{Design of ionizable cationic lipids and clinical applications}

The initial reason for employing ionizable cationic lipids was to achieve a system where efficient encapsulation of negatively charged polymers could be achieved at low $\mathrm{pH}$, but which also exhibited a relatively uncharged surface at $\mathrm{pH}$ 7.4. However, it soon became clear that the potency of LNPsiRNA systems for silencing genes in hepatocytes following i.v. administration was sensitive to the species of ionizable cationic lipid employed. Thus, a comprehensive screening process was undertaken to see whether further improvements over the gene silencing achieved by LNP-siRNA systems in the liver (hepatocytes) were possible. A guiding hypothesis was that the most potent ionizable cationic lipids would satisfy two constraints. The first constraint is that the pKa of the lipid should be low enough to prevent the LNP from having a high positive surface charge at physiological $\mathrm{pH}$ values. High surface charge is associated with toxicity and rapid clearance from the circulation by the fixed and free macrophages. The second constraint is that the $\mathrm{pKa}$ should be high enough that the ionizable cationic lipid can adopt a positively charged form at acidic endosomal $\mathrm{pH}$ values. This is so that cationic lipids can combine with endogenous endosomal anionic lipids to promote membrane lytic nonbilayer structures such as the hexagonal $\mathrm{H}_{\mathrm{II}}$ phase, resulting in intracellular delivery (Fig. 2). It is well known that cationic lipids combine with anionic lipids to adopt nonbilayer structures and lipids that adopt such structures have significant membrane-destabilizing properties [33,51,52]. A related design principle was that the cationic lipid should be relatively unsaturated as it is well established that unsaturated lipids adopt these nonbilayer structures more readily $[53,54]$.

The polymorphic phase tendencies of lipids can be rationalized according to the "shape" hypothesis [54] where a comparison of the cross-sectional area of the lipid head group to the cross-sectional area of the lipid tails determines the macrostructure formed upon hydration (Fig. 3). Lipids such as lysolipids and PEG-conjugated lipids with a "coneshaped" geometry prefer to form micellar structures. Alternatively, lipids such as distearylphosphatidylcholine (DSPC) have a cylindrical geometry compatible with bilayer structures. Other lipids with small uncharged head groups and unsaturated acyl chains, such as DOPE, display an "invertedcone" geometry, and preferentially form the inverted hexagonal $\left(\mathrm{H}_{\mathrm{II}}\right)$ phase [53].

Using a murine Factor VII (FVII) model for hepatic gene silencing, LNPs containing a variety of ionizable cationic lipids were evaluated to determine the effective dose to achieve $50 \%$ gene silencing $\left(\mathrm{ED}_{50}\right)$. The rational-design process first identified 2,2-dilinoleyl-4-(2-dimethylaminoethyl)-[1,3]-dioxolane (DLin-KC2-DMA, or KC2) as an ionizable cationic lipid that had improved activity compared to DLinDMA [8]. Further optimization of the LNP-siRNA systems was achieved by increasing the lipid content to $50 \mathrm{~mol} \%$ [8], resulting in an $\mathrm{ED}_{50}$ for $\mathrm{KC} 2$ of $0.1 \mathrm{mg} s i R N A / \mathrm{kg}$ with no apparent hepatic toxicities. A subsequent screening study identified heptatriaconta6,9,28,31-tetraen-19-yl-4-(dimethylamino)butanoate (DLinMC3-DMA or MC3) [7]. The structures of the ionizable cationic lipids discussed are shown in Fig. 1. The optimized lipid formulation of MC3/cholesterol/DSPC/PEG-lipid (50/ $38.5 / 10 / 1.5 \mathrm{~mol} \%$ ) in the same FVII assay displayed an $\mathrm{ED}_{50}$ of $0.005 \mathrm{mg} / \mathrm{kg}$.

MC3 represents the most potent ionizable cationic lipid thus far identified for hepatic gene silencing. It was determined that lipids such as MC3 and KC2 exhibited optimal apparent $\mathrm{pK}_{\mathrm{a}}$ values in the region of 6.5. This $\mathrm{pK}_{\mathrm{a}}$ activity 


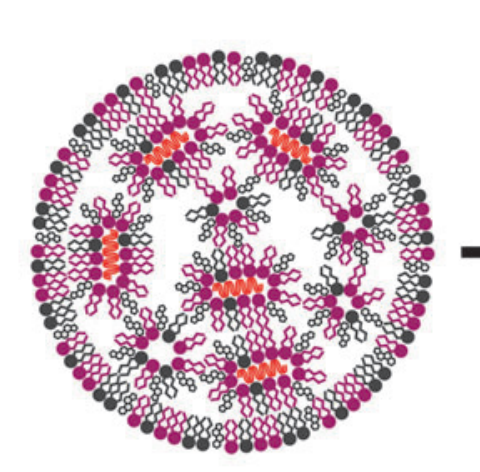

(2)
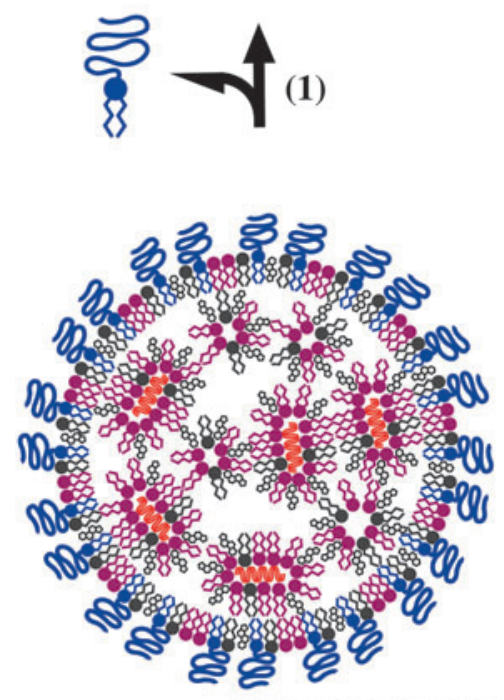

Extracellular space
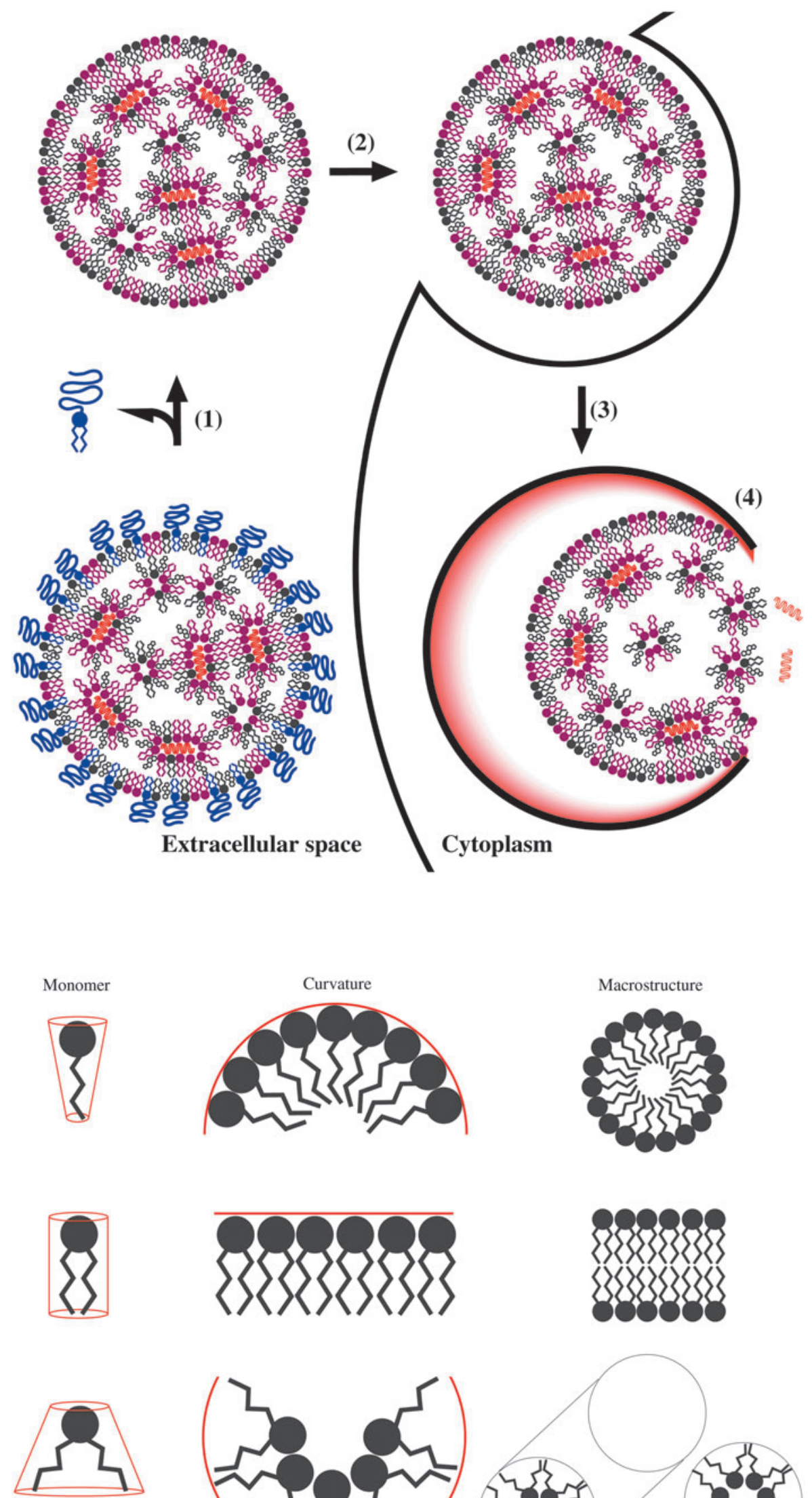
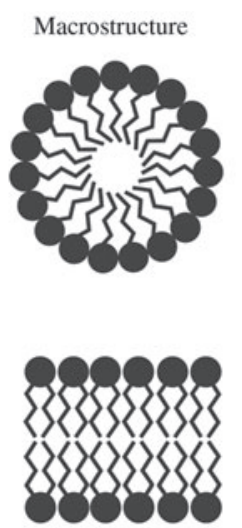

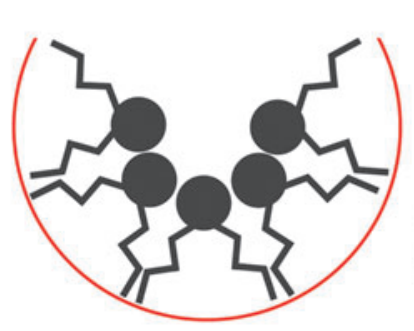

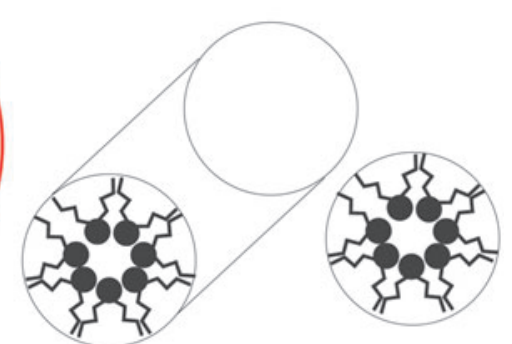

Hexagonal phase

Inverted micelle
FIG. 2. Mechanism of transfection with an LNP formulation of siRNA. LNP transfection following endocytosis is mediated by an endosomolytic event where the contents of the nanoparticle are delivered to the cytosol of the target cell. (1) As mentioned previously, in order for the LNP to become transfection competent, it requires the PEG-lipid to diffuse from the particle into the lipid sink. (2) Shedding of the PEG-lipid from the surface of the particle triggers internalization into the cell. (3) Following endocytosis, the acidification of the endosome results in an LNP with a strong positive charge on the surface. (4) Interactions between the LNP and endosomal membrane result in a membrane fusion event that leads to cytosolic delivery of the payload. LNP, lipid nanoparticle; siRNA, short interfering RNA.
FIG. 3. The molecular shape hypothesis and lipid polymorphic behavior. The molecular shape hypothesis suggests that the macrostructure adopted by a lipid is dictated by the geometry of the lipid. This geometry is defined by the cross-sectional area of the lipid head group and cross-sectional area of the lipid tails. (Top) A cone-shaped lipid (large head group, small tail area) generates positive curvature in membrane, and in isolation will form micelles. (Middle) A cylindricalshaped lipid does not generate curvature, and in isolation will form bilayer structures. (Bottom) A lipid with an inverted cone geometry induces negative curvature and upon hydration forms the hexagonal $\left(\mathrm{H}_{\mathrm{II}}\right)$ phase, inverted micelles, and cubic phases (not shown). 


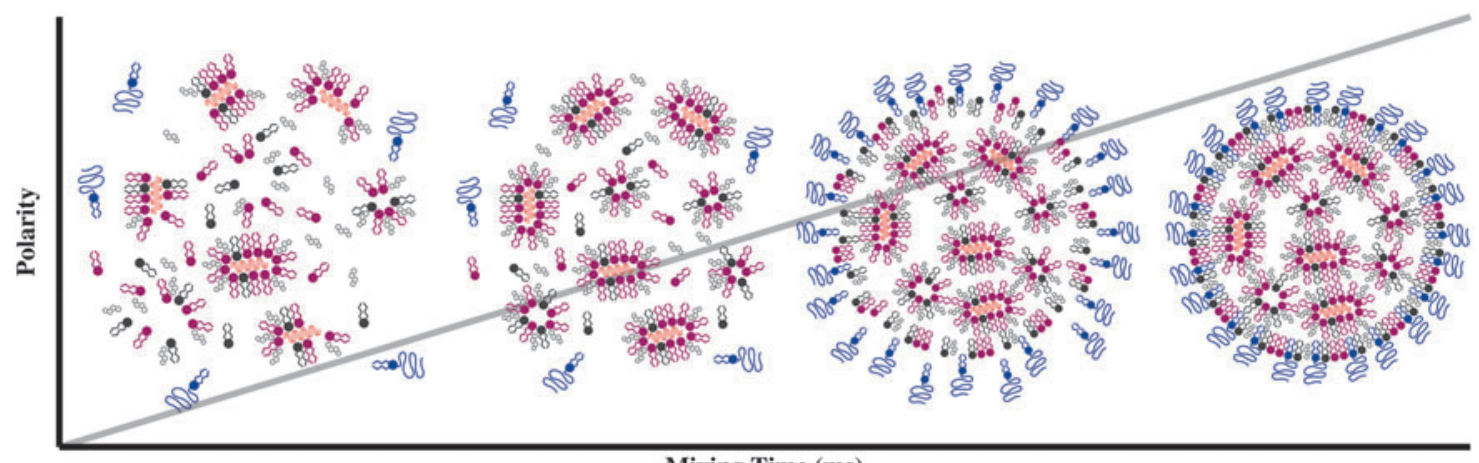

Mixing Time (ms)

FIG. 4. Proposed mechanism of LNP formation and nanostructured core. Increase in solvent polarity (light gray line, background) drives the self-assembly of LNP-siRNA formulations. LNP-siRNA are hypothesized to form an electron-dense core structure as a result of significant lipid and nucleic acid present in the internal compartment. (Left) The first interactions to occur upon mixing of the ethanol and aqueous streams are those between the ionizable cationic lipids and the negatively charged nucleic acids. (Middle left) As the solvent polarity progressively increases, the hydrophobic inverted micellar structures coalesce generating the core of the LNP. (Middle right) As mixing continues, the more polar lipids (such as PEGlipid and DSPC) coat the surface of the nanoprecipitates. (Right) The resulting part has an electron-dense core structure surrounded by a lipid monolayer. Ionizable cationic lipid (purple), phospholipid (dark gray), PEG-lipid (violet), cholesterol (light gray), and siRNA (red). DSPC, distearylphosphatidylcholine.

correlation was confirmed by mixing ionizable lipids with different $\mathrm{pKa}$ values to achieve net $\mathrm{pKa}$ values ranging from 5.6 to 6.9. LNP-siRNA containing lipids with a net $\mathrm{pKa}$ of $\sim 6.5$ displayed optimal silencing in the murine FVII model [7]. Overall, it should be noted that optimization of ionizable cationic lipids led to a truly remarkable improvement in gene silencing potency. Initial LNP-siRNA systems composed of DLinDAP exhibited an $\mathrm{ED}_{50}$ of $40-50 \mathrm{mg} / \mathrm{kg}$, while the $\mathrm{ED}_{50}$ for MC3-LNP was $0.005 \mathrm{mg} / \mathrm{kg}$, an improvement of more than three orders of magnitude with no increase in toxicity. This corresponds to a therapeutic index improvement greater than 8,000-fold, suggesting clinical utility.

As a result, LNP-siRNA systems containing MC3 [7,55,56] rapidly transitioned into clinical trials conducted by Alnylam Pharmaceuticals to treat transthyretin (TTR)-induced amyloidosis, a hereditary disease resulting from mutations in the TTR gene, leading to deposition of TTR fibrils in cardiac and nerve tissue. TTR is synthesized predominantly in hepatocytes and thus susceptible to the gene silencing possible with LNPsiRNA systems containing MC3. A Phase I clinical evaluation of the formulation indicated that the LNP-siRNA was well tolerated at doses up to $0.5 \mathrm{mg}$ siRNA/ $\mathrm{kg}$ [57]. A subsequent phase II study determined that only $0.3 \mathrm{mg}$ siRNA/ $\mathrm{kg}$ body weight in a dosing regimen of Q3 W was required to achieve robust gene silencing, with an excellent tolerability profile [58]. It should be noted that as lipid-based drug delivery systems have been associated with adverse infusion-related immune events [59], a mixture of immune suppressants is given before LNP-siRNA [58]. Patisiran recently (September 2017) completed a Phase III clinical trial that met all primary and secondary endpoints [9] and exhibited less toxicity than the control substance (sterilized normal saline). A New Drug Application for regulatory approval of Patisiran was made to the FDA in December 2017.

\section{Structure of LNP-siRNA formulations}

LNP-siRNA systems containing KC2 and MC3 exhibited an electron-dense core structure as visualized by cryo-TEM [60] compared to the clearly lamellar structure of certain LNP systems containing plasmid [31] or ASO [39]. This electrondense core structure has been suggested to result from the initial formation of inverted micellar structures of cationic lipids surrounding siRNA molecules that are subsequently coated with polar lipids, such as the PEG and phospholipids, as the polarity of the medium increases $[43,60]$ (Fig. 4). This proposed LNP structure has been supported by a number of findings such as the dependence of particle size on PEG-lipid content, ${ }^{31} \mathrm{P}-\mathrm{NMR}$ studies showing immobilization of the nucleic acid, the "currant bun" morphology of entrapped negatively charged gold nanoparticles, and computer simulations. These findings and their contribution to the suggested structure are briefly discussed in turn:

(1) It was determined by varying the concentration of PEG-lipid (at the expense of cholesterol) that particle diameters could be varied from $120 \mathrm{~nm}$ (at $0.25 \mathrm{~mol} \%$ PEG-lipid) to $25 \mathrm{~nm}$ (at $5 \mathrm{~mol} \%$ PEG-lipid) [43]. These observations were consistent with the PEGlipid being present in the surface monolayer and thus dictating LNP size by influencing the surface-to-core lipid ratio.

(2) "Limit size" refers to the smallest structures compatible with a particular lipid composition. The formation of limit-size particles was achieved through formulation of $\mathrm{KC} 2 / \mathrm{PEG}$-lipid (90/10 mol\%) with siRNA generating $23 \mathrm{~nm}$ particles [60]. This size corresponds to the theoretical size of a single inverted micellar structure coated with a surface monolayer of lipids [60];

(3) ${ }^{31} \mathrm{P}-\mathrm{NMR}$ data of phosphorothioate-modified siRNA (to prevent overlap from DSPC-phosphate signal) showed a loss of signal when siRNA was entrapped in LNP systems, showing that the inverted micellar structures immobilize the siRNA on the NMR timescale [60];

(4) The entrapment of gold nanoparticles through a different charge density provided a "tracker" for analysis by cryo-TEM due to the electron density differences. These formulations displayed a "currant 
bun" morphology, which strongly supports the inverted micellar hypothesis [61];

(5) Computer simulations of siRNA interactions with ionizable cationic lipids suggested periodic compartments in which the siRNA could be immobilized [60];

\section{LNP-siRNA protein adsorption and endogenous targeting to hepatocytes}

The relative specificity of LNP-siRNA systems containing ionizable cationic lipid for hepatocytes suggested that a targeting mechanism is operating. Given previous results [62] demonstrating that clearance of neutral liposomes to hepatocytes is mediated by ApoE, its role in delivery of LNPsiRNA systems was investigated. These studies demonstrated that LNP-siRNA gene silencing activity was significantly decreased in an ApoE knockout mouse model $\left(\mathrm{ApoE}^{-/-}\right)$and activity could be rescued by preincubating the particles with ApoE before administration [63]. It was therefore concluded that ApoE association with LNP-siRNA systems plays a major role in triggering LNP uptake into hepatocytes. It appears that the LDL receptor plays a role in the ApoEdependent uptake. In a low-density lipoprotein receptor knockout model $\left(\mathrm{LDLR}^{-/-}\right.$), LNP-siRNA formulations displayed less gene silencing activity (leading to higher $\mathrm{ED}_{50}$ values) than in wild-type animals [63]. LNP activity could be rescued through addition of a multivalent targeting ligand, $\mathrm{N}$ acetylgalactosamine (GalNAc), for the hepatocyte asialoglycoprotein receptor, thereby promoting internalization through an alternative endocytic route.

ApoE-binding to LNP-siRNA systems subsequent to the dissociation of the PEG-lipid coating clearly plays a role in the rapid particle accumulation in the liver following i.v. administration [64]. A study with a variety of LNP-siRNA formulations showed that $95 \%$ of the injected dose of LNP with the composition ionizable lipid/cholesterol/DSPC/PEGlipid (50/38.5/10/1.5 mol\%) accumulated in the liver within $15 \mathrm{~min}$ [64]. The size of the LNP system also plays a role in determining potency with LNP with diameter $\sim 80 \mathrm{~nm}$ exhibiting maximum gene silencing potency [64]. Smaller sizes ( $\sim 25 \mathrm{~nm}$ diameter) achieved by increasing the PEG-lipid content to $5 \mathrm{~mol} \%$ resulted in significantly reduced gene silencing activity using the FVII model. The $\mathrm{ED}_{50}$ increased from $0.05 \mathrm{mg}$ siRNA $/ \mathrm{kg}$ for the $80 \mathrm{~nm}$ diameter to $0.8 \mathrm{mg}$ siRNA/kg for the $25 \mathrm{~nm}$ diameter LNP-siRNA system. This reduced activity was attributed [64] to instability of the smaller particles leading to dissociation of the cationic lipid from the particle. The reduced activity could be partially recovered by increasing the ionizable cationic lipid content to $60 \mathrm{~mol} \%$ and increasing the ionizable lipid to siRNA charge ratio $(\mathrm{N} / \mathrm{P})$ from 3 to 6 , resulting in an improvement of the $\mathrm{ED}_{50}$ from $0.8 \mathrm{mg} \mathrm{siRNA} / \mathrm{kg}$ to $0.3 \mathrm{mg} / \mathrm{kg}$ [64].

\section{Future Perspectives}

Clinical validation of LNP-siRNA systems to silence genes such as TTR in hepatocytes clearly opens the door to using similar systems to silence other genes in hepatocytes such as proprotein convertase subtilisin/kexin type 9 (PCSK9) for cholesterol-lowering therapy [65]. Important additional opportunities include the use of LNP systems to silence genes in tissues other than the liver and the extension of LNP technology to delivering mRNA and CRSPR-Cas for gene expression and gene editing purposes. The ability to extend gene silencing to nonhepatic tissues will dramatically extend the range of gene therapies possible, including treating diseases such as cancer by silencing oncogenes. LNPmRNA systems, on the other hand, offer considerable potential for expressing therapeutic proteins to treat a wide variety of diseases requiring protein replacement as well as vaccine applications. Finally, the ability to practice gene editing in vivo would clearly enable treatment of many hereditary diseases. A number of these opportunities are now being evaluated in clinical settings (Table 1).

\section{Design of LNP-siRNA for extrahepatic targets}

Liposomal systems used for the delivery of small-molecule therapeutics often employ a persistent PEG-lipid coat to increase circulation times, resulting in improved delivery to nonhepatic tissues such as tumor sites [12]. However, incorporation of persistent PEG-lipids such as PEG-DSG achieved the desired circulation times for LNP-siRNA systems [64], but dramatically reduced transfection competency, and as observed with a number of cationic lipid systems, can completely abolish it $[66,67]$. Thus, while extrahepatic distributions of LNP containing persistent PEG-lipids can be achieved, the LNP reaching these tissues have limited transfection potency. This "PEG-dilemma" has yet to be solved.

The situation is further complicated by the dual role of the PEG-lipid in LNP-siRNA formulations, serving to control particle size [43] as well as circulation lifetimes in vivo [64]. LNP-siRNA systems containing 1.5\% PEG-DSG exhibit a size of $45 \mathrm{~nm}$, but display rapid clearance $\left(\mathrm{t}_{1 / 2}<15 \mathrm{~min}\right)$ and reasonable gene silencing potency, presumably because they retain the ability to associate with $\mathrm{ApoE}$ at these relatively low PEG contents [64]. In contrast, LNPs containing $5 \mathrm{~mol} \%$ PEG-DSG and a size of $25 \mathrm{~nm}$ exhibit $\mathrm{t}_{1 / 2}$ of $\sim 10-12 \mathrm{~h} \mathrm{[64].}$ The LNP size can also influence circulation time in another way; particles with $0.5 \mathrm{~mol} \%$ PEG-DSG and a size of $80 \mathrm{~nm}$ have a longer circulation $\mathrm{t}_{1 / 2}$ of 3-4 $\mathrm{h}$ [64], presumably because these larger systems are less able to pass through the fenestration of liver vasculature, leading to longer circulation lifetimes [64]. Higher (5 mol\%) PEG-DSG levels leading to small particles of $25 \mathrm{~nm}$ stay in circulation for longer as they have a high PEG density precluding adsorption of serum proteins such as ApoE [64].

LNP-siRNA systems containing PEG-DSG (3-5 mol\%; $30 \mathrm{~nm}$ diameter) that exhibit long circulation lifetimes ( $\mathrm{t}_{1 / 2}$ of 10-12 $\mathrm{h}$ ) have been used to knock down the androgen receptor (AR), a fundamental driver of prostate cancer, in an LNCaP xenograft model [68]. However, the dose of LNPsiRNA required to achieve appreciable gene silencing was $30 \mathrm{mg} / \mathrm{kg}$, more than 6,000 times higher than required to achieve an $\mathrm{ED}_{50}$ in hepatocytes. In an effort to improve potency, a targeting ligand for the prostate-specific membrane antigen was incorporated, resulting in improved gene silencing activity [68], but not the orders of magnitude improvement required for clinical potential. Similarly, knockdown activity and tumor growth delay were reported at distal tumors in an enzalutamide-resistant $\mathrm{LNCaP}$ xenograft model when LNP-siRNA against clusterin was used in combination with AR-ASO [69]. The aggressive dosing 
regimen in these studies resulted in a cumulative dose of 30 and $50 \mathrm{mg}$ siRNA/ $\mathrm{kg}$, respectively. While these doses were tolerated in preclinical studies, the dose levels employed were close to maximum tolerated doses, leading to a small therapeutic index that does not warrant clinical development.

In the central nervous system, ApoE is mainly produced by astrocytes [70] and transports cholesterol to neurons through ApoE receptors, which are members of the low-density lipoprotein receptor gene family. Thus, LNP-siRNA systems identical to those employed for silencing genes in hepatocytes can be used to silence genes in neurons. However, nanoparticles such as LNP-siRNA do not passively cross the blood-brain barrier, requiring local administration. The first demonstration of LNP-siRNA activity in neurons was targeting the GluN1 subunit of the $N$-methyl-D-Aspartate receptor following intracortical and intracerebroventricular administration [71]. Intracortical administration resulted in silencing around the injection site, while intracerebroventricular administration resulted in more widespread knockdown. Subsequent studies with LNP-siRNA enabled the elucidation of a secondary role of the ion exchanger SLC26A11 as a voltage-gated ion channel involved in neuronal swelling and cytotoxic edema [72]. Suppression of SLC26A11 by LNP-siRNA-mediated knockdown dramatically decreased neuronal swelling. These studies highlight the potential of LNP-siRNA for target identification studies, as well as the potential for therapeutic applications for severe disorders where direct administration through intracortical, interventricular, or intrathecal routes is possible.

\section{Design of LNP for gene expression and editing}

The initial design of LNP systems (SPLP) for larger nucleic acid polymers was for delivery of pDNA to express a therapeutic gene. As noted previously, low expression levels and an inefficient and nonscalable formulation technique precluded further development. Following the development of the ethanol loading technique for encapsulating siRNA into LNP, it was shown that this protocol could also be used to encapsulate mRNA, pDNA, and other negatively charged macromolecules $[61,73]$. This has led to significant advances to enable mRNA-based therapeutics. Extensive work has been conducted to develop ionizable cationic lipids optimized for mRNA cargoes [74], leading to improvements in gene expression in hepatocytes following i.v. administration that are some 20-fold higher than observed for LNP mRNA systems containing MC3. Intravenous administration of LNPs containing unmodified, sequence-engineered mRNA encoding for erythropoietin (EPO) that was administered intravenously at a single dose of $1.3 \mathrm{mg}$ mRNA in pigs $(20 \mathrm{~kg})$ and a dose of $100 \mu \mathrm{g}$ mRNA in cynomolgus monkeys resulted in high systemic EPO levels, increased reticulocyte numbers, and elevation of the hematocrit [75]. This has led to the possibility of using the liver as a bioreactor to express therapeutic proteins. A compelling example is the demonstration that i.v. administration of LNP-mRNA encoding VRC01 (a broadly neutralizing antibody against HIV-1) results in high levels of the VRC01 antibody in the circulation for 7 days in humanized mice. Such levels were sufficient to afford complete protection against a challenge with the SF162 strain of HIV $24 \mathrm{~h}$ later. This protection required as little as $15 \mu \mathrm{g}$ mRNA per animal [76].
LNP-mRNA systems also show considerable potential for vaccine applications. Initial work in mice showed that LNPmRNA formulations at doses of $5 \mu \mathrm{g}$ nucleoside-modified mRNA administered through subcutaneous, intradermal, or intramuscular injection could result in sustained expression of luciferase for up to 10 days, whereas i.v. administration resulted in a burst of expression followed by rapid decrease over 5 days [77]. A significant body of data has been published on the use of mRNA for vaccine applications [78]; certain studies employing LNP systems are of particular interest $[76,79,80]$. LNP-mRNA formulations encoding the premembrane envelope protein prM-E derived from the Zika virus were tested in mice and rhesus macaques and showed that a dose of only $50 \mu \mathrm{g}$ mRNA was required to protect against a Zika virus challenge [80]. Kranz et al. have demonstrated the application of lipid-based delivery of RNA to dendritic cells for cancer immunotherapy [81]. It was shown that i.v. administration of RNA lipoplexes resulted in antigen expression in lymphoid-resident DCs. In addition, lipoplexes containing RNA encoding viral or mutant neoantigens or endogenous self-antigens induced strong antitumor responses in mouse models of melanoma, colorectal cancer, and lung cancer [81]. The safety and tolerability of the RNA lipoplexes encoding four tumor antigens (NY-ESO-1, MAGE-A3, tyrosinase, and TPTE) are currently under evaluation in a Phase I clinical trial (NCT02410733, Table 1).

With regard to LNP formulations of pDNA, the application of the ionizable cationic lipid approach is leading to potent transfection systems, but the lipid compositions for achieving maximum transfection differ substantially from those employed for siRNA. For example, KC2-based LNP demonstrated significantly better in vitro transfection than MC3-based formulations [82], and unsaturated "helper" lipids such as dioleoylphosphatidylcholine, stearoyl-oleoyl-phosphatidylcholine, and DOPE improved transfection potency. Optimized LNP-pDNA formulations showed robust, mosaic transfection (with no detectable toxicities) of the developing chicken limb-bud following direct in ovo injection [82].

CRISPR-based genome editing has generated much excitement due to the prospect of potentially permanently curing genetic disorders [83]. However, the safe and efficient nonviral delivery of the gene-editing components (Cas protein and a single guide RNA, sgRNA) in vivo have proven to be a considerable challenge so far [5,84]. Most recently, Yin et al. developed LNP for delivery of Cas9 mRNA and sgRNAs (LNP-CRISPR) targeting the mouse PCSK9 gene for treatment of hypercholesterolemia [85]. A single injection of LNP-CRISPR in mice resulted in undetectable PCSK9 serum levels, over $80 \%$ editing in the liver and a reduction of total cholesterol by $35 \%-40 \%$ [85]. Results of a 12-month study in mice with LNP-CRISPR for editing of the TTR gene were recently announced by Intellia Therapeutics. Results showed $\sim 97 \%$ maintained reduction in serum TTR following a single dose and around $70 \%$ editing at the target DNA site in hepatocytes [86].

\section{Summary}

The discovery of ionizable cationic lipids has proven to be a critical factor in the development of clinically viable LNPsiRNA treatments. Potent lipids such as MC3 allow efficient encapsulation of siRNA, result in a relatively neutral LNP 
surface charge in the circulation, and are able to promote escape of the siRNA into the cytosol following endocytosis. With the likely FDA approval of the first LNP-siRNA formulation for treatment of TTR-mediated amyloidosis in 2018, there are high clinical expectations for this new class of drugs for silencing disease-causing genes in the liver and other tissues. In addition, LNP containing ionizable cationic lipids are facilitating the rapid development of other gene therapies based on mRNA and CRISPR/Cas. Important issues remain, such as cost-effectiveness, toxicity, and off-target effects; however, the fact that LNP formulations of genetic drugs can now facilitate the knockdown, expression, or editing of virtually any gene in hepatocytes, with the promise of extension to other tissues, constitutes a major breakthrough in the development of (personalized) gene therapies.

\section{Acknowledgments}

The work of JAK and PRC is funded by a foundation grant (FDN 148469) from the Canadian Institutes of Health Research (CIHR). RvdM is supported by a VENI Fellowship (\# 14385) from the Netherlands Organization for Scientific Research (NWO).

\section{Author Disclosure Statement}

No competing financial interests exist.

\section{References}

1. Cullis PR and MJ Hope. (2017). Lipid nanoparticle systems for enabling gene therapies. Mol Ther 25:1467-1475.

2. Hajj KA and KA Whitehead. (2017). Tools for translation: non-viral materials for therapeutic mRNA delivery. Nat Rev Mater 2:17056.

3. Kanasty R, JR Dorkin, A Vegas and D Anderson. (2013). Delivery materials for siRNA therapeutics. Nat Mater 12:967.

4. Kotterman MA and DV Schaffer. (2014). Engineering adeno-associated viruses for clinical gene therapy. Nat Rev Genet 15:445-451.

5. Liu C, L Zhang, H Liu and K Cheng. (2017). Delivery strategies of the CRISPR-Cas9 gene-editing system for therapeutic applications. J Control Release 266:17-26.

6. Alnylam ${ }^{\circledR}$ Pharmaceuticals, Inc. (2017). In Media, A. (ed.). BusinessWire, www.businesswire.com/news/home/ 20170920005628/en Accessed January 11, 2018.

7. Jayaraman M, SM Ansell, BL Mui, YK Tam, J Chen, X Du, D Butler, L Eltepu, S Matsuda, et al. (2012). Maximizing the potency of siRNA lipid nanoparticles for hepatic gene silencing in vivo. Angew. Chem Int Ed Engl 51:8529-8533.

8. Semple SC, A Akinc, J Chen, AP Sandhu, BL Mui, CK Cho, DW Sah, D Stebbing, EJ Crosley, et al. (2010). Rational design of cationic lipids for siRNA delivery. Nat Biotechnol 28:172-176.

9. Alnylam ${ }^{\circledR}$ Pharmaceuticals, Inc. (2017). http://investors .alnylam.com/news-releases/news-release-details/alnylamand-sanofi-present-positive-complete-results-apollo Accessed January 11, 2018.

10. Alnylam ${ }^{\circledR}$ Pharmaceuticals, Inc. (2017). http://investors .alnylam.com/news-releases/news-release-details/alnylamcompletes-submission-new-drug-application-us-food-and

11. Alnylam ${ }^{\circledR}$ Pharmaceuticals, Inc. (2018). http://investors .alnylam.com/news-releases/news-release-details/alnylamannounces-ema-acceptance-marketing-authorisation
12. Allen TM and PR Cullis. (2013). Liposomal drug delivery systems: from concept to clinical applications. Adv Drug Deliv Rev 65:36-48.

13. Kawabata K, Y Takakura and M Hashida. (1995). The fate of plasmid DNA after intravenous injection in mice: involvement of scavenger receptors in its hepatic uptake. Pharm Res 12:825-830.

14. Judge AD, V Sood, JR Shaw, D Fang, K McClintock and I MacLachlan. (2005). Sequence-dependent stimulation of the mammalian innate immune response by synthetic siRNA. Nat Biotechnol 23:457-462.

15. Layzer JM, AP McCaffrey, AK Tanner, Z Huang, MA Kay and BA Sullenger. (2004). In vivo activity of nucleaseresistant siRNAs. RNA 10:766-771.

16. Choi HS, W Liu, P Misra, E Tanaka, JP Zimmer, B Itty Ipe, MG Bawendi and JV Frangioni. (2007). Renal clearance of quantum dots. Nat Biotechnol 25:1165-1170.

17. Choi HS, W Liu, F Liu, K Nasr, P Misra, MG Bawendi and JV Frangioni. (2009). Design considerations for tumourtargeted nanoparticles. Nat Nanotechnol 5:42.

18. Geary RS, TA Watanabe, L Truong, S Freier, EA Lesnik, NB Sioufi, H Sasmor, M Manoharan and AA Levin. (2001). Pharmacokinetic properties of 2'-O-(2-methoxyethyl)modified oligonucleotide analogs in rats. J Pharmacol Exp Ther 296:890-897.

19. Sands H, LJ Gorey-Feret, AJ Cocuzza, FW Hobbs, D Chidester and GL Trainor. (1994). Biodistribution and metabolism of internally $3 \mathrm{H}$-labeled oligonucleotides. I. Comparison of a phosphodiester and a phosphorothioate. Mol Pharmacol 45:932-943.

20. Shen X and DR Corey. (2018). Chemistry, mechanism and clinical status of antisense oligonucleotides and duplex RNAs. Nucleic Acids Res 46:1584-1600.

21. Mirzayans R, MV Middlestadt and MC Paterson. (1992). Cytotoxic and mutagenic effects of methylnitrosourea in two human fetal fibroblast strains differing in O6methylguanine-DNA methyltransferase activity. Carcinogenesis 13:1185-1190.

22. Capecchi MR. (1980). High efficiency transformation by direct microinjection of DNA into cultured mammalian cells. Cell 22:479-488.

23. Felgner PL, TR Gadek, M Holm, R Roman, HW Chan, M Wenz, JP Northrop, GM Ringold and M Danielsen. (1987). Lipofection: a highly efficient, lipid-mediated DNAtransfection procedure. Proc Natl Acad Sci U S A 84:74137417.

24. Ernsting MJ, M Murakami, A Roy and SD Li. (2013). Factors controlling the pharmacokinetics, biodistribution and intratumoral penetration of nanoparticles. J Control Release 172:782-794.

25. Fraley R, S Subramani, P Berg and D Papahadjopoulos. (1980). Introduction of liposome-encapsulated SV40 DNA into cells. J Biol Chem 255:10431-10435.

26. Fraley R, RM Straubinger, G Rule, EL Springer and D Papahadjopoulos. (1981). Liposome-mediated delivery of deoxyribonucleic acid to cells: enhanced efficiency of delivery related to lipid composition and incubation conditions. Biochemistry 20:6978-6987.

27. Filion MC and NC Phillips. (1997). Toxicity and immunomodulatory activity of liposomal vectors formulated with cationic lipids toward immune effector cells. Biochim Biophys Acta 1329:345-356.

28. Lappalainen K, I Jaaskelainen, K Syrjanen, A Urtti and S Syrjanen. (1994). Comparison of cell proliferation and 
toxicity assays using two cationic liposomes. Pharm Res 11:1127-1131.

29. Wheeler JJ, L Palmer, M Ossanlou, I MacLachlan, RW Graham, YP Zhang, MJ Hope, P Scherrer and PR Cullis. (1999). Stabilized plasmid-lipid particles: construction and characterization. Gene Ther 6:271-281.

30. Fenske DB, I MacLachlan and PR Cullis. (2002). Stabilized plasmid-lipid particles: a systemic gene therapy vector. Methods Enzymol 346:36-71.

31. Tam P, M Monck, D Lee, O Ludkovski, EC Leng, K Clow, H Stark, P Scherrer, RW Graham and PR Cullis. (2000). Stabilized plasmid-lipid particles for systemic gene therapy. Gene Ther 7:1867.

32. Ambegia E, S Ansell, P Cullis, J Heyes, L Palmer and I MacLachlan. (2005). Stabilized plasmid-lipid particles containing PEG-diacylglycerols exhibit extended circulation lifetimes and tumor selective gene expression. Biochim Biophys Acta 1669:155-163.

33. Hafez IM, N Maurer and PR Cullis. (2001). On the mechanism whereby cationic lipids promote intracellular delivery of polynucleic acids. Gene Ther 8:1188-1196.

34. Aramaki Y, S Takano and S Tsuchiya. (1999). Induction of apoptosis in macrophages by cationic liposomes. FEBS Lett 460:472-476.

35. Dokka S, D Toledo, X Shi, V Castranova and Y Rojanasakul. (2000). Oxygen radical-mediated pulmonary toxicity induced by some cationic liposomes. Pharm Res 17:521-525.

36. Yan W, W Chen and L Huang. (2008). Reactive oxygen species play a central role in the activity of cationic liposome based cancer vaccine. J Control Release 130:22-28.

37. Bailey AL and PR Cullis. (1994). Modulation of membrane fusion by asymmetric transbilayer distributions of amino lipids. Biochemistry 33:12573-12580.

38. Semple SC, SK Klimuk, TO Harasym, N Dos Santos, SM Ansell, KF Wong, N Maurer, H Stark, PR Cullis, et al. (2001). Efficient encapsulation of antisense oligonucleotides in lipid vesicles using ionizable aminolipids: formation of novel small multilamellar vesicle structures. Biochim Biophys Acta 1510:152-166.

39. Maurer N, KF Wong, H Stark, L Louie, D McIntosh, T Wong, P Scherrer, SC Semple and PR Cullis. (2001). Spontaneous entrapment of polynucleotides upon electrostatic interaction with ethanol-destabilized cationic liposomes. Biophys J 80:2310-2326.

40. Jeffs LB, LR Palmer, EG Ambegia, C Giesbrecht, S Ewanick and I MacLachlan. (2005). A scalable, extrusionfree method for efficient liposomal encapsulation of plasmid DNA. Pharm Res 22:362-372.

41. Batzri S and ED Korn. (1973). Single bilayer liposomes prepared without sonication. Biochim Biophys Acta 298: 1015-1019.

42. Hirota S, CT de Ilarduya, LG Barron and FC Szoka, Jr. (1999). Simple mixing device to reproducibly prepare cationic lipid-DNA complexes (lipoplexes). Biotechniques 27:286-290.

43. Belliveau NM, J Huft, PJ Lin, S Chen, AK Leung, TJ Leaver, AW Wild, JB Lee, RJ Taylor, et al. (2012). Microfluidic synthesis of highly potent limit-size lipid nanoparticles for in vivo delivery of siRNA. Mol Ther Nucleic Acids 1:e37.

44. Evers MJW, JA Kulkarni, R van der Meel, PR Cullis, P Vader and RM Schiffelers. (2018). State-of-the-art design and rapid-mixing production techniques of lipid nanoparticles for nucleic acid delivery. Small Methods Submitted.
45. de Jong S, G Chikh, L Sekirov, S Raney, S Semple, S Klimuk, N Yuan, M Hope, P Cullis and Y Tam. (2007). Encapsulation in liposomal nanoparticles enhances the immunostimulatory, adjuvant and anti-tumor activity of subcutaneously administered CpG ODN. Cancer Immunol Immunother 56:1251-1264.

46. Raney SG, KD Wilson, L Sekirov, G Chikh, SD de Jong, PR Cullis and YK Tam. (2008). The effect of circulation lifetime and drug-to-lipid ratio of intravenously administered lipid nanoparticles on the biodistribution and immunostimulatory activity of encapsulated CpG-ODN. J Drug Target 16:564-577.

47. Wilson KD, SG Raney, L Sekirov, G Chikh, SD deJong, PR Cullis and YK Tam. (2007). Effects of intravenous and subcutaneous administration on the pharmacokinetics, biodistribution, cellular uptake and immunostimulatory activity of $\mathrm{CpG}$ ODN encapsulated in liposomal nanoparticles. Int Immunopharmacol 7:1064-1075.

48. Zimmermann TS, ACH Lee, A Akinc, B Bramlage, D Bumcrot, MN Fedoruk, J Harborth, JA Heyes, LB Jeffs, et al. (2006). RNAi-mediated gene silencing in non-human primates. Nature 441:111.

49. Tam YY, S Chen and PR Cullis. (2013). Advances in lipid nanoparticles for siRNA delivery. Pharmaceutics 5:498-507.

50. Judge A, K McClintock, JR Phelps and I Maclachlan. (2006). Hypersensitivity and loss of disease site targeting caused by antibody responses to PEGylated liposomes. Mol Ther 13:328-337.

51. Ellens H, J Bentz and FC Szoka. (1985). H+- and Ca2+induced fusion and destabilization of liposomes. Biochemistry 24:3099-3106.

52. Cullis PR and MJ Hope. (1978). Effects of fusogenic agent on membrane structure of erythrocyte ghosts and the mechanism of membrane fusion. Nature 271:672.

53. Cullis PR and B de Kruijff. (1978). The polymorphic phase behaviour of phosphatidylethanolamines of natural and synthetic origin. A 31P NMR study. Biochim Biophys Acta 513: $31-42$.

54. Gruner SM, PR Cullis, MJ Hope and CP Tilcock. (1985). Lipid polymorphism: the molecular basis of nonbilayer phases. Annu Rev Biophys Biophys Chem 14:211-238.

55. Butler JS, A Chan, S Costelha, S Fishman, JL Willoughby, TD Borland, S Milstein, DJ Foster, P Goncalves, et al. (2016). Preclinical evaluation of RNAi as a treatment for transthyretin-mediated amyloidosis. Amyloid 23:109-118.

56. Alnylam ${ }^{\circledR}$ Pharmaceuticals. I. (2012). http://investors .alnylam.com/news-releases/news-release-details/alnylaminitiates-phase-i-clinical-study-aln-ttr02-rnai Accessed March 14, 2018.

57. Coelho T, D Adams, A Silva, P Lozeron, PN Hawkins, T Mant, J Perez, J Chiesa, S Warrington, et al. (2013). Safety and efficacy of RNAi therapy for transthyretin amyloidosis. New Engl J Med 369:819-829.

58. Suhr OB, T Coelho, J Buades, J Pouget, I Conceicao, J Berk, H Schmidt, M Waddington-Cruz, JM Campistol, et al. (2015). Efficacy and safety of patisiran for familial amyloidotic polyneuropathy: a phase II multi-dose study. Orphanet J Rare Dis 10:109.

59. Szebeni J, F Muggia, A Gabizon and Y Barenholz. (2011). Activation of complement by therapeutic liposomes and other lipid excipient-based therapeutic products: prediction and prevention. Adv Drug Deliv Rev 63:1020-1030.

60. Leung AK, IM Hafez, S Baoukina, NM Belliveau, IV Zhigaltsev, E Afshinmanesh, DP Tieleman, CL Hansen, MJ Hope and PR Cullis. (2012). Lipid nanoparticles containing 
siRNA synthesized by microfluidic mixing exhibit an electron-dense nanostructured core. J Phys Chem C Nanomater Interfaces 116:18440-18450.

61. Leung AK, YY Tam, S Chen, IM Hafez and PR Cullis. (2015). Microfluidic mixing: a general method for encapsulating macromolecules in lipid nanoparticle systems. J Phys Chem B 119:8698-8706.

62. Yan X, F Kuipers, LM Havekes, R Havinga, B Dontje, K Poelstra, GL Scherphof and JA Kamps. (2005). The role of apolipoprotein $\mathrm{E}$ in the elimination of liposomes from blood by hepatocytes in the mouse. Biochem Biophys Res Commun 328:57-62.

63. Akinc A, W Querbes, S De, J Qin, M Frank-Kamenetsky, KN Jayaprakash, M Jayaraman, KG Rajeev, WL Cantley, et al. (2010). Targeted delivery of RNAi therapeutics with endogenous and exogenous ligand-based mechanisms. Mol Ther 18:1357-1364.

64. Chen S, YYC Tam, PJC Lin, MMH Sung, YK Tam and PR Cullis. (2016). Influence of particle Size on the in vivo potency of lipid nanoparticle formulations of siRNA. J Control Release 235:236-244.

65. Fitzgerald K, S White, A Borodovsky, BR Bettencourt, A Strahs, V Clausen, P Wijngaard, JD Horton, J Taubel, et al. (2017). A highly durable RNAi therapeutic inhibitor of PCSK9. N Engl J Med 376:41-51.

66. Harvie P, FMP Wong and MB Bally. (2000). Use of poly(ethylene glycol)-lipid conjugates to regulate the surface attributes and transfection activity of lipid-DNA particles. J Pharm Sci 89:652-663.

67. Song LY, QF Ahkong, Q Rong, Z Wang, S Ansell, MJ Hope and B Mui. (2002). Characterization of the inhibitory effect of PEG-lipid conjugates on the intracellular delivery of plasmid and antisense DNA mediated by cationic lipid liposomes. Biochim Biophys Acta 1558:1-13.

68. Lee JB, K Zhang, YY Tam, J Quick, YK Tam, PJ Lin, S Chen, Y Liu, JK Nair, et al. (2016). A Glu-urea-Lys ligand-conjugated lipid nanoparticle/siRNA system inhibits androgen receptor expression in vivo. Mol Ther Nucleic Acids 5:e348.

69. Yamamoto Y, PJ Lin, E Beraldi, F Zhang, Y Kawai, J Leong, H Katsumi, L Fazli, R Fraser, et al. (2015). siRNA lipid nanoparticle potently silences clusterin and delays progression when combined with androgen receptor cotargeting in enzalutamide-resistant prostate cancer. Clin Cancer Res 21:4845-4855.

70. Vance JE and H Hayashi. (2010). Formation and function of apolipoprotein E-containing lipoproteins in the nervous system. Biochim Biophys Acta 1801:806-818.

71. Rungta RL, HB Choi, PJ Lin, RW Ko, D Ashby, J Nair, M Manoharan, PR Cullis and BA Macvicar. (2013). Lipid nanoparticle delivery of siRNA to silence neuronal gene expression in the brain. Mol Ther Nucleic Acids 2:e136.

72. Rungta RL, HB Choi, JR Tyson, A Malik, L DissingOlesen, PJC Lin, SM Cain, PR Cullis, TP Snutch and BA MacVicar. (2015). The cellular mechanisms of neuronal swelling underlying cytotoxic edema. Cell 161:610-621.

73. Kulkarni JA, YYC Tam, S Chen, J Zaifman, YK Tam, PR Cullis and S Biswas. (2017). Rapid synthesis of lipid nanoparticles containing hydrophobic inorganic nanoparticles. Nanoscale 9:13600-13609.

74. Acuitas Therapeutics. (2018). https://acuitastx.com/portfoliotype/lipid-nanoparticles Accessed January 11, 2018.

75. Thess A, S Grund, BL Mui, MJ Hope, P Baumhof, M FotinMleczek and T Schlake. (2015). Sequence-engineered
mRNA without chemical nucleoside modifications enables an effective protein therapy in large animals. Mol Ther 23: 1456-1464.

76. Pardi N, AJ Secreto, X Shan, F Debonera, J Glover, Y Yi, H Muramatsu, H Ni, BL Mui, et al. (2017). Administration of nucleoside-modified mRNA encoding broadly neutralizing antibody protects humanized mice from HIV-1 challenge. Nat Commun 8:14630.

77. Pardi N, S Tuyishime, H Muramatsu, K Kariko, BL Mui, YK Tam, TD Madden, MJ Hope and D Weissman. (2015). Expression kinetics of nucleoside-modified mRNA delivered in lipid nanoparticles to mice by various routes. J Control Release 217:345-351.

78. Pardi N, MJ Hogan, FW Porter and D Weissman. (2018). mRNA vaccines - a new era in vaccinology. Nat Rev Drug Discov [Epub ahead of print]; DOI: 10.1038/nrd.2017.243.

79. Lutz J, S Lazzaro, M Habbeddine, KE Schmidt, P Baumhof, BL Mui, YK Tam, TD Madden, MJ Hope, et al. (2017). Unmodified mRNA in LNPs constitutes a competitive technology for prophylactic vaccines. NPJ Vaccines 2:29.

80. Pardi N, MJ Hogan, RS Pelc, H Muramatsu, H Andersen, CR DeMaso, KA Dowd, LL Sutherland, RM Scearce, et al. (2017). Zika virus protection by a single low-dose nucleosidemodified mRNA vaccination. Nature 543:248.

81. Kranz LM, M Diken, H Haas, S Kreiter, C Loquai, KC Reuter, M Meng, D Fritz, F Vascotto, et al. (2016). Systemic RNA delivery to dendritic cells exploits antiviral defence for cancer immunotherapy. Nature 534:396-401.

82. Kulkarni JA, JL Myhre, S Chen, YYC Tam, A Danescu, JM Richman and PR Cullis. (2016). Design of lipid nanoparticles for in vitro and in vivo delivery of plasmid DNA. Nanomedicine 13:1377-1387.

83. Singh V, D Braddick and PK Dhar. (2017). Exploring the potential of genome editing CRISPR-Cas9 technology. Gene 599:1-18.

84. Schaefer KA, WH Wu, DF Colgan, SH Tsang, AG Bassuk and VB Mahajan. (2017). Unexpected mutations after CRISPR-Cas9 editing in vivo. Nat Methods 14:547-548.

85. Yin H, CQ Song, S Suresh, Q Wu, S Walsh, LH Rhym, E Mintzer, MF Bolukbasi, LJ Zhu, et al. (2017). Structure-guided chemical modification of guide RNA enables potent non-viral in vivo genome editing. Nat Biotechnol 35:1179-1187.

86. Finn JD, AR Smith, MC Patel, L Shaw, MR Youniss, J van Heteren, T Dirstine, C Ciullo, R Lescarbeau, et al. A single administration of CRISPR/Cas9 lipid nanoparticles achieves robust and persistent in vivo genome editing. Cell Rep 22: 2227-2235.

Address correspondence to: Jayesh A. Kulkarni, BSc (Hons) Department of Biochemistry and Molecular Biology University of British Columbia 2350 Health Sciences Mall Vancouver V6T $1 Z 3$ British Columbia Canada

E-mail: j.kulkarni@alumni.ubc.ca

Received for publication January 26, 2018; accepted after revision March 20, 2018. 